\title{
Gradient and Amplitude Scattering in Surface-Corrugated Waveguides
}

\author{
F. M. Izrailev \\ Instituto de Física, \\ N. M. Makarov $\dagger$ \\ Instituto de Ciencias, \\ M. Rendón \\ Facultad de Ciencias de la Electrónica, \\ Universidad Autónoma de Puebla, \\ Puebla, Pue., 72570, México
}

(Dated: November 9, 2018)

\begin{abstract}
We investigate the interplay between amplitude and square-gradient scattering from the rough surfaces in multi-mode waveguides (conducting quantum wires). The main result is that for any (even small in height) roughness the square-gradient terms in the expression for the wave scattering length (electron mean free path) are dominant, provided the correlation length of the surface disorder is small enough. This important effect is missed in existing studies of the surface scattering.
\end{abstract}

1. The problem of wave transport (both classical and quantum) through the guiding surface-disordered systems has a quite long history and remains a hot topic

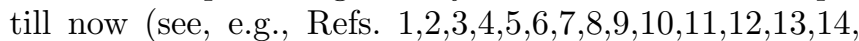
15, 16 and references therein). One of the main tools to treat this problem is the reduction of the wave/electron surface scattering to the bulk one in such a way that the latter can be described by an effective Hamiltonian with a complicated potential, however, with flat boundaries. Applying this approach, one can reasonably discriminate between the so-called amplitude and gradient scattering, and analyze their interplay explicitly.

To the best of our knowledge, originally the idea of this approach was discussed by Migda ${ }^{5}$. After, it was frequently used in the theories of classical and quantum wave/electron scattering, see, e.g., Refs. 6 7 8 9 10 1112, 13. But in the majority of them ${ }^{6,7.8 .9,10}$ the study was restricted to the lowest order in the roughness height $\sigma$.

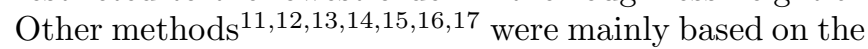
principal assumption that the surface roughness is sufficiently smooth.

In this contribution we present the theory of wave scattering from rough surfaces, which takes into account both the amplitude and gradient scattering mechanisms. The important point is that we do not assume any special restrictions on the model parameters except for general conditions of weak scattering. The latter provides us with an appropriate perturbative approach in scattering potential, however, is not restricted by the smoothness of surfaces.

The main attention is paid to the competition between the amplitude and gradient scattering. One of our main results is that at fixed r.m.s. roughness height $\sigma$, the less correlation length $R_{c}$ of a random surface profile, the larger contribution of the gradient mechanism. Thus, by passing from the smooth to white-noise profiles, the gradient scattering becomes to prevail. We have analyzed this crossover and obtained the estimates describing the transition to the dominating gradient scattering. In par- ticular, we show that this transition is located within the region of small roughness slopes where $\sigma / R_{c} \ll 1$.

2. In what follows we consider an open plane waveguide (or conducting quasi-one-dimensional quantum wire) of the average width $d$, stretched along the $x$-axis. For simplicity, one (lower) surface of the waveguide is assumed to be flat, $z=0$, while the other (upper) surface has a rough profile $z=w(x)=d+\sigma \xi(x)$ with $\langle\xi(x)\rangle=0$ and $\left\langle\xi^{2}(x)\right\rangle=1$. The average $\langle\ldots\rangle$ is performed over different realizations of a statistically homogeneous and isotropic Gaussian random function $\xi(x)$. We also assume that its pair correlator $\left\langle\xi(x) \xi\left(x^{\prime}\right)\right\rangle=\mathcal{W}\left(x-x^{\prime}\right)$ decreases on a scale $R_{c}$, with the normalization $\mathcal{W}(0)=1$. The roughness-height power (RHP) spectrum $W\left(k_{x}\right)$ is defined by

$$
W\left(k_{x}\right)=\int_{-\infty}^{\infty} d x \exp \left(-i k_{x} x\right) \mathcal{W}(x) .
$$

Since $\mathcal{W}(x)$ is an even function of $x$, its Fourier transform (11) is even, real and nonnegative function of $k_{x}$. The RHP spectrum has maximum at $k_{x}=0$ with $W(0) \sim R_{c}$, and decreases on the scale $R_{c}^{-1}$.

In order to analyze the surface scattering for our model, we employ the method of the retarded Green's function $\mathcal{G}\left(x, x^{\prime} ; z, z^{\prime}\right)$. Specifically, we start with the Dirichlet boundary-value problem

$$
\begin{aligned}
& \left(\frac{\partial^{2}}{\partial x^{2}}+\frac{\partial^{2}}{\partial z^{2}}+k^{2}\right) \mathcal{G}\left(x, x^{\prime} ; z, z^{\prime}\right) \\
& =\delta\left(x-x^{\prime}\right) \delta\left(z-z^{\prime}\right), \\
& \mathcal{G}\left(x, x^{\prime} ; z=0, z^{\prime}\right)=\mathcal{G}\left(x, x^{\prime} ; z=w(x), z^{\prime}\right)=0 .
\end{aligned}
$$

Here the wave number $k$ is equal to $\omega / c$ for an electromagnetic wave of the frequency $\omega$ and TE polarization, propagating through a waveguide with perfectly conducting walls. As for an electron quantum wire, $k$ is the Fermi wave number within the isotropic Fermi-liquid model. In 
order to express the surface scattering as a bulk one, we perform the transformation to new coordinates,

$$
x_{\text {new }}=x_{\text {old }}, \quad z_{\text {new }}=z_{\text {old }} d /[d+\sigma \xi(x)],
$$

in which both waveguide surfaces are flat. Correspondingly, we introduce the canonically conjugate Green's function, $\mathcal{G}_{\text {new }}=d^{-1} \sqrt{w(x) w\left(x^{\prime}\right)} \mathcal{G}_{\text {old }}$ and omit the subscript "new" in what follows. As a result, we arrive at the equivalent boundary-value problem governed by the equation with a "bulk" perturbation potential,

$$
\begin{aligned}
& \left\{\frac{\partial^{2}}{\partial x^{2}}+\frac{\partial^{2}}{\partial z^{2}}+k^{2}-\left[1-\frac{d^{2}}{w^{2}(x)}\right] \frac{\partial^{2}}{\partial z^{2}}\right. \\
& -\frac{\sigma}{w(x)}\left[\xi^{\prime}(x) \frac{\partial}{\partial x}+\frac{\partial}{\partial x} \xi^{\prime}(x)\right]\left[\frac{1}{2}+z \frac{\partial}{\partial z}\right] \\
& \left.+\frac{\sigma^{2} \xi^{\prime 2}(x)}{w^{2}(x)}\left[\frac{3}{4}+3 z \frac{\partial}{\partial z}+z^{2} \frac{\partial^{2}}{\partial z^{2}}\right]\right\} \mathcal{G}\left(x, x^{\prime} ; z, z^{\prime}\right) \\
& =\delta\left(x-x^{\prime}\right) \delta\left(z-z^{\prime}\right) \\
& \mathcal{G}\left(x, x^{\prime} ; z=0, z^{\prime}\right)=\mathcal{G}\left(x, x^{\prime} ; z=d, z^{\prime}\right)=0 .
\end{aligned}
$$

Here the prime stands for the derivative over $x$.

We emphasize that Eq. (5) is exact and valid for any form of $w(x)$. As one can see, the scattering potential depends both on the roughness profile $\sigma \xi(x)$ and on its gradient $\sigma \xi^{\prime}(x)$. Moreover, the potential contains the term with the square gradient $\sigma^{2} \xi^{\prime 2}(x)$. This term is proportional to $\sigma^{2}$ and for this reason was neglected in all previous studies of transport properties in the surfacedisordered waveguides. However, as a matter of fact, the square gradient introduces the operator $\hat{\mathcal{V}}(x)=\xi^{\prime 2}(x)-$ $\left\langle\xi^{\prime 2}(x)\right\rangle$, which plays a special role. Its pair correlator,

$$
\left\langle\hat{\mathcal{V}}(x) \hat{\mathcal{V}}\left(x^{\prime}\right)\right\rangle=2\left\langle\xi^{\prime}(x) \xi^{\prime}\left(x^{\prime}\right)\right\rangle^{2}=2 \mathcal{W}^{\prime \prime 2}\left(x-x^{\prime}\right)
$$

determines the square-gradient power (SGP) spectrum

$$
T\left(k_{x}\right)=\int_{-\infty}^{\infty} d x \exp \left(-i k_{x} x\right) \mathcal{W}^{\prime \prime 2}(x)
$$

One should stress that although by integration by parts the power spectrum of the roughness gradients $\sigma \xi^{\prime}(x)$ can be reduced to the RHP spectrum $W\left(k_{x}\right)$, this is not possible for the SGP spectrum $T\left(k_{x}\right)$. This very fact reflects a highly non-trivial role of the square-gradient scattering, giving rise to the competition with the well known amplitude scattering, in spite of the seeming smallness of the term $\sigma^{2} \xi^{\prime 2}(x)$.

To proceed, we pass from Eq. (5) to the Dyson-type equation, performing the ensemble averaging with the use of the technique developed in Ref. 3. The method allows one to develop the consistent perturbative approach with respect to the scattering potential, which takes adequately into account the multiple scattering from the corrugated boundary. After quite cumbersome calculations we have obtained the average Green's function which in the normal-mode representation has the form

$$
\begin{aligned}
& \left\langle\mathcal{G}\left(x, x^{\prime} ; z, z^{\prime}\right)\right\rangle=\sum_{n=1}^{N_{d}} \sin \left(\frac{\pi n z}{d}\right) \sin \left(\frac{\pi n z^{\prime}}{d}\right) \\
& \times \frac{\exp \left(i k_{n}\left|x-x^{\prime}\right|\right)}{i k_{n} d} \exp \left(-\frac{\left|x-x^{\prime}\right|}{2 L_{n}}\right) .
\end{aligned}
$$

Here $k_{n}=\sqrt{k^{2}-(\pi n / d)^{2}}$ corresponds to the unperturbed lengthwise wave number $k_{x}$, and $N_{d}=[k d / \pi]$ is the number of propagating modes (or conducting electron channels) determined by the integer part [...] of the ratio $k d / \pi$.

3. Our interest is in the attenuation length or total mean free path $L_{n}$ of the $n$-th mode. Its inverse value is given by the imaginary part of the proper self-energy and, in accordance with the form of the scattering potential, consists of two terms describing different scattering mechanisms,

$$
\frac{1}{L_{n}}=\frac{1}{L_{n}^{(1)}}+\frac{1}{L_{n}^{(2)}} .
$$

The first length $L_{n}^{(1)}$ is determined by the expression

$$
\begin{aligned}
\frac{1}{L_{n}^{(1)}}= & \sigma^{2} \frac{(\pi n / d)^{2}}{k_{n} d} \sum_{n^{\prime}=1}^{N_{d}} \frac{\left(\pi n^{\prime} / d\right)^{2}}{k_{n^{\prime}} d} \\
& \times\left[W\left(k_{n}+k_{n^{\prime}}\right)+W\left(k_{n}-k_{n^{\prime}}\right)\right] .
\end{aligned}
$$

Its diagonal term is formed by the amplitude scattering while the off-diagonal terms result from the gradient one. Eq. (11) coincides with that previously obtained by different methods (see, e.g., Ref. 1).

The second length $L_{n}^{(2)}$ is associated solely with the square-gradient mechanism due to the operator $\hat{\mathcal{V}}(x)$,

$$
\frac{1}{L_{n}^{(2)}}=\sum_{n^{\prime}=1}^{N_{d}} \frac{1}{L_{n n^{\prime}}^{(2)}}
$$

Its diagonal term controls the intramode scattering,

$$
\begin{aligned}
\frac{1}{L_{n n}^{(2)}}= & \frac{\sigma^{4}}{2} \frac{(\pi n / d)^{4}}{k_{n}^{2}}\left[\frac{1}{3}+\frac{1}{(2 \pi n)^{2}}\right]^{2} \\
& \times\left[T\left(2 k_{n}\right)+T(0)\right]
\end{aligned}
$$

The off-diagonal partial length $L_{n \neq n^{\prime}}^{(2)}$ describes the $i n$ termode scattering (from $n$ to $n^{\prime} \neq n$ channels),

$$
\begin{aligned}
\frac{1}{L_{n \neq n^{\prime}}^{(2)}}= & \frac{8 \sigma^{4}}{\pi^{4}} \frac{(\pi n / d)^{2}}{k_{n}} \frac{\left(\pi n^{\prime} / d\right)^{2}}{k_{n^{\prime}}} \frac{\left(n^{2}+n^{\prime 2}\right)^{2}}{\left(n^{2}-n^{\prime 2}\right)^{4}} \\
& \times\left[T\left(k_{n}+k_{n^{\prime}}\right)+T\left(k_{n}-k_{n^{\prime}}\right)\right] .
\end{aligned}
$$

To the best of our knowledge, in the surface-scattering problem for multi-mode waveguides the operator $\hat{\mathcal{V}}(x)$ 
was never taken into account, and, as a result, the squaregradient attenuation length $L_{n}^{(2)}$ was missed in previous studies.

Let us analyze the conditions under which Eqs. (10) - (14) are derived. We stress that the Dyson-type equation for the average Green's function was obtained within the second-order approximation in the perturbation potential. This means that the self-energy in this equation contains the binary correlator of the surface-scattering potential and the unperturbed Green's function. In terms of the diagrammatic technique this is similar to the "simple vortex" or, the same, Bourret approximation ${ }^{18}$. Following the ideas discussed in Ref. 2, one can show that this approximation is justified when the broadening $1 / 2 L_{n}$ of the quantum wave number $k_{n}$ is much less than both the correlation scale $R_{c}^{-1}$ and the spacing $\left|k_{n}-k_{n \pm 1}\right| \approx\left|\partial k_{n} / \partial n\right|$ between neighboring quantum wave numbers. The same conditions also arise due to another approximation which is the use of the unperturbed value $k_{n}$ in the expression for the self-energy, instead of the perturbed one. Now we take into account that $\left|\partial k_{n} / \partial n\right| \sim \Lambda_{n}^{-1}$, where $\Lambda_{n}$ is the distance between two successive reflections of a wave from the rough boundary inside the $n$-th channel. As a result, we come to the following conditions of a weak surface scattering

$$
\Lambda_{n}=2 k_{n} d /(\pi n / d) \ll 2 L_{n}, \quad R_{c} \ll 2 L_{n} .
$$

These inequalities imply that the electron/wave weakly attenuates on both the correlation length $R_{c}$ and the $c y$ cle length $\Lambda_{n}$.

As one can see, the expressions (11) and (12) - (14) represent, respectively, basic contributions from principally different surface-scattering mechanisms related to the amplitude, gradient and square-gradient terms. It should be emphasized that the corrections proportional to $\sigma^{4}$, originated from higher order approximations in the amplitude and gradient terms of the perturbation potential, are smaller than the main contribution (11) under the conditions (15). Contrary, the square-gradient terms give rise to the $\sigma^{4}$-terms in Eqs.(13)-(14) which should not be neglected due to a specific dependence on the correlation length $R_{c}$. Note that Eq. (15) implicitly includes the requirement for the surface corrugations be small in height $(\sigma \ll d)$, but does not restrict the value $\sigma / R_{c}$ of the roughness slope.

4. Since $L_{n}^{(1)}$ and $L_{n}^{(2)}$ depend on as many as four dimensionless parameters $(k \sigma)^{2}, k R_{c}, k d / \pi$, and $n$, the complete analysis appears to be quite complicated. For this reason, below we restrict ourselves by the analysis of the interplay between $L_{n}^{(1)}$ and $L_{n}^{(2)}$ as a function of the dimensionless correlation length $k R_{c}$ for $N_{d} \approx k d / \pi \gg 1$.

As follows from Eq. (11), the inverse value of the amplitude-scattering length typically increases with an increase of $k R_{c}$. Specifically, in the case of the smallscale roughness $\left(k R_{c} \ll 1 \lesssim k \Lambda_{n}\right)$ we have $1 / L_{n}^{(1)} \propto k R_{c}$. Then, within the intermediate region where $1 \ll k R_{c} \ll$ $k \Lambda_{n}$, the increase of $1 / L_{n}^{(1)}$ slows down, or can even be replaced by the decrease for some values of the model parameters. Finally, for large-scale roughness and strong correlations $\left(1 \lesssim k \Lambda_{n} \ll k R_{c}\right)$ the value of $1 / L_{n}^{(1)}$ again starts to increase linearly with $k R_{c}$.

In contrast with $1 / L_{n}^{(1)}$, the inverse square-gradient scattering length $1 / L_{n}^{(2)}$ reveals a monotonous decrease as the parameter $k R_{c}$ increases. At small $\left(k R_{c} \ll 1 \lesssim k \Lambda_{n}\right)$ and extremely large $\left(1 \lesssim k \Lambda_{n} \ll k R_{c}\right)$ values of $k R_{c}$, this decrease obeys the law $1 / L_{n}^{(2)} \propto\left(k R_{c}\right)^{-3}$, due to $T(0) \sim R_{c}^{-3}$.

¿From this analysis it becomes clear that the curves displaying $1 / L_{n}^{(1)}$ and $1 / L_{n}^{(2)}$ must intersect, and one can observe the crossover from the square-gradient to amplitude surface scattering. To the left from the crossing point $\left(k R_{c}\right)_{\odot}$ the square-gradient scattering length prevails, $L_{n}^{(2)} \ll L_{n}^{(1)}$. To its right the main contribution is due to the well known amplitude scattering, $L_{n}^{(1)} \ll L_{n}^{(2)}$. If the crossing point falls onto the interval of the smallscale roughness $\left(k R_{c} \ll 1\right)$, its dependence on the model parameters is described by

$$
\left(k R_{c}\right)_{\odot}^{2} \sim(k \sigma) n / \sqrt{k_{n} d} .
$$

This estimate shows that the crossing point is smaller for smaller values of the dimensionless roughness height $k \sigma$, as well as for smaller mode indices $n$, or for larger values of the parameter $k d / \pi$.

In Fig. 11 we display the dependence of $\Lambda_{n} / 2 L_{n}$ as a function of $k R_{c}$ assuming the Gaussian binary correlator $\mathcal{W}(x)=\exp \left(-x^{2} / 2 R_{c}^{2}\right)$ for random surface profile $\xi(x)$. The curves are plotted starting from such values of $k R_{c}$ for which $\Lambda_{n} / 2 L_{n}^{(2)}=1$, according to the first condition of Eq. (15). Taking into account the second condition restricting the maximal value of $k R_{c}$, we plot every curve within the range where $R_{c}<2 L_{n}^{(1)}$. As one can see, all curves have the crossover from the square-gradient to amplitude surface scattering. The first (lowest) one with $(k \sigma)^{2}=10^{-4}$ has the crossing point $\left(k R_{c}\right)_{\odot} \approx 0.2$ located within the interval of small-scale roughness, and the crossover reveals a small dip centered at $\left(k R_{c}\right)_{\odot}$. The curve obeys the asymptotic behavior $\left(k R_{c}\right)^{-3}$ to the left from $\left(k R_{c}\right)_{\odot}$ due to the main contribution from $\Lambda_{n} / 2 L_{n}^{(2)}$. Then the quantity $\Lambda_{n} / 2 L_{n}^{(1)}$ becomes dominating in the sum (10), therefore, the curve starts to rise. Firstly, the linear dependence on $k R_{c}$ on the right deep-side (where $k R_{c}<1$ ) is replaced with a smoother one (for $k R_{c}>1$ ). Finally, for $R_{c}>\Lambda_{n}$ (strong correlations) the linear dependence restores.

The crossing points of the second, third and fourth curves have the values of the order one. Here the total attenuation length $L_{n}$ within the whole small-scale region is formed by the square-gradient scattering length $L_{n}^{(2)}$. In full agreement with Eq. (16) the presented curves display that the smaller parameter $(k \sigma)^{2}$ the smaller value of the crossing point $\left(k R_{c}\right)_{\odot}$.

Note that for all curves in Fig.1 the roughness height is small, $\sigma / d \ll 1$. Furthermore, for the amplitude- 


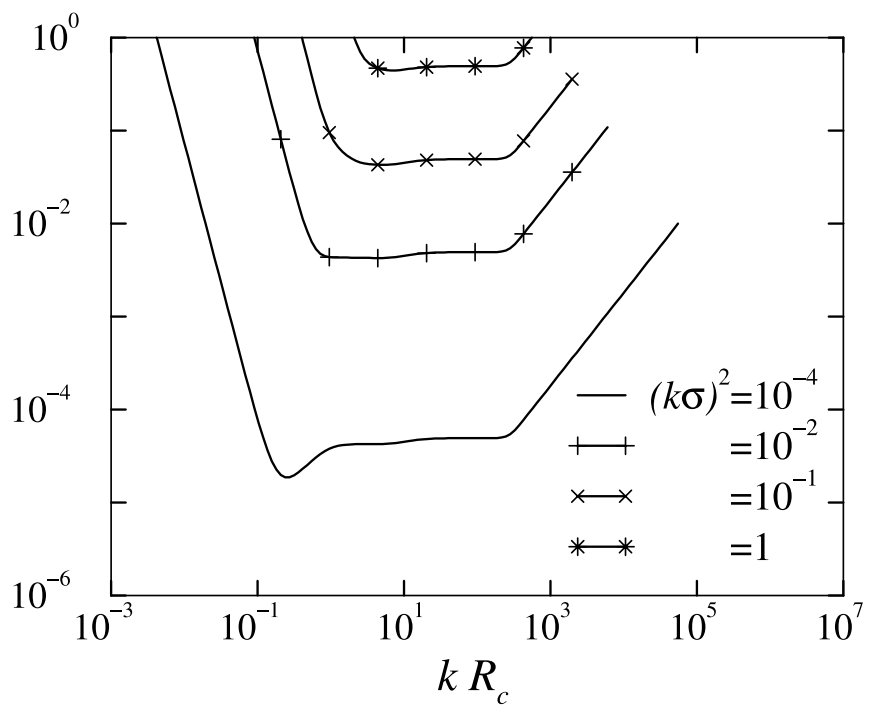

FIG. 1: $\Lambda_{n} / 2 L_{n}$ versus $k R_{c}$ at $k d / \pi=62.5, n=31$ and different $(k \sigma)^{2}$.

dominated scattering (to the right from the point $\left(k R_{c}\right)_{\odot}$ where $\Lambda_{n} / 2 L_{n}^{(1)}$ mainly contributes), the average corrugation slope is also small for all data, $\sigma / R_{c} \ll 1$. The roughness slope remains to be small at the crossing points too, but increases to their left with the decrease of $k R_{c}$.
As a result, to the left from the crossing point where the square-gradient term $\Lambda_{n} / 2 L_{n}^{(2)}$ prevails, the slope reaches the values of the order one, or even larger for the first tree curves.

In conclusion, we have discovered the principal importance of the square-gradient surface-scattering mechanism which was never taken into account in the literature. We have shown that at any fixed value of the roughness height $\sigma$ one can indicate the region of small values of the correlation length $R_{c}$ where the new squaregradient scattering length $L_{n}^{(2)}$ predominates over the known amplitude scattering length $L_{n}^{(1)}\left(L_{n}^{(2)} \ll L_{n}^{(1)}\right)$. The predominance occurs in spite of the fact that $1 / L_{n}^{(1)}$ is proportional to $\sigma^{2}$ while $1 / L_{n}^{(2)}$ is proportional to $\sigma^{4}$. This happens since the two lengths are determined by the substantially different roughness-height $W\left(k_{x}\right)$ and roughness-square-gradient $T\left(k_{x}\right)$ power spectra, that have vastly different dependencies on $R_{c}$. It is remarkable that the square-gradient mechanism prevails in the commonly used region $k R_{c} \ll 1$ of a small-scale boundary perturbation, where the surface roughness is typically described via the white-noise potential.

This research was partially supported by the CONACYT (México) grant No 43730, and by the VIEP-BUAP (México) under the grant II 104-05/ING/G.
* Electronic address: izrailev@venus.ifuap.buap.mx

† Electronic address: makarov@siu.buap.mx

¥ Electronic address: mrendon@venus.ifuap.buap.mx

1 F. G. Bass, I. M. Fuks, Wave Scattering from Statistically Rough Surfaces (Pergamon, New York, 1979).

2 S. M. Rytov, Yu. A. Kravtsov, and V. I. Tatarskii, Principles of Statistical Radiophysics (Springer, Berlin, 1989).

3 A. R. McGurn and A. A. Maradudin, Phys. Rev. B 30, 3136 (1984).

4 J. A. Sánchez-Gil, V. Freilikher, I. V. Yurkevich, and A. A. Maradudin, Phys. Rev. Lett. 80, 948 (1998); J. A. Sánchez-Gil, V. Freilikher, A. A. Maradudin, and I. V. Yurkevich, Phys. Rev. B 59, 5915 (1999).

5 A. B. Migdal, Qualitative Methods in Quantum Theory (Benjamin, London, 1977). p. 98.

6 J. A. Konrady, J. Acoust. Soc. Am. 56, 1687 (1974).

7 Z. Tešanović, M. Jarić, S. Maekawa, Phys. Rev. Lett. 57, 2760 (1986).

8 N. Trivedi and N. W. Ashcroft, Phys. Rev. B 38, 12298 (1988).

9 A. E. Meyerovich and S. Stepaniants, Phys. Rev. Lett. 73, 316 (1994); Phys. Rev. B 51, 17116 (1995); Phys. Rev. B
58, 13242 (1998); Phys. Rev. B 60, 9129 (1999); J. Phys.: Condens. Matter 12, 5575 (2000).

10 G. A. Luna-Acosta, Kyungsun Na, L. E. Reichl, A. Krokhin, Phys. Rev. E 53, 3271 (1996).

11 A. B. Isers, A. A. Puzenko, I. M. Fuks, Akust. Zh. 36, 454 (1990) [Sov. Phys. Acoust. 36, 253 (1990)]; Journal of Electromagnetic Waves and Applications 5, 1419 (1991).

12 N. M. Makarov and Yu. V. Tarasov, J.Phys.: Condens. Matter 10, 1523 (1998); Phys. Rev. B 64, 235306 (2001).

13 G. A. Luna-Acosta, J. A. Méndez-Bermúdez, F. M. Izrailev, Phys. Rev. E 64, 036206 (2001).

14 A. G. Voronovich, Wave Scattering from Rough Surfaces (Springer, Berlin, 1994).

15 A. A. Krokhin, N. M. Makarov, V. A. Yampol'skii, J. Phys.: Condens. Matter 3, 4621 (1991).

16 N. M. Makarov, A. V. Moroz, V. A. Yampol'skii, Phys. Rev. B 52, 6087 (1995).

17 V. I. Tatarskii, Waves in Random Media 3, 127 (1993); ibid 7, 557 (1997); ibid 10, 339 (2000).

18 R. C. Bourret, Nuovo Cimento 26, 1 (1962). 